\title{
Could a mannequin simplify rhinopharyngeal swab collection in COVID 19 patients?
}

\author{
Francesca Boscolo Nata ${ }^{1}$ (D) Barbara Pedruzzi ${ }^{1} \cdot$ Emanuele Ferri $^{1} \cdot$ Francesco Scotto di Clemente $^{1}$. \\ Giovanni Campolieti ${ }^{1} \cdot$ Fabio Manoni $^{2} \cdot$ Giovanni Cristalli $^{1}$
}

Received: 19 May 2020 / Accepted: 4 June 2020 / Published online: 9 June 2020

○) Springer-Verlag GmbH Germany, part of Springer Nature 2020, corrected publication 2023

\begin{abstract}
Rhinopharyngeal swab collection is used to test patients for COVID-19; unfortunately, the false negative rate is around 30\% in symptomatic patients, and maybe even higher in asymptomatic ones. A correct swab collection is consequently critical. Swabs are usually performed by dedicated personnel, but at the present moment, the methods for its training are not standardized. In a Letter to the Editor recently published in the European Archives of Otorhinolaringology, Tagliabue et al. describe the training method used in their Institution: the personnel dedicated to swab collection increases its confidence with the procedure by observing endoscopic anatomy, while an otolaryngologist performs rigid endoscopy. Although we found this paper interesting, we think that the author's proposal has timing and financial drawbacks that should be considered in daily activity, especially in an emergency period like the pandemic we are experiencing. In this Letter to the Editor, we discuss some pre-analytic and analytic issues that should be considered while performing rhinopharyngeal swabs, and we propose the use of a mannequin to train personnel, thus reducing the risk of infection for health workers, and patient's discomfort.
\end{abstract}

Keywords COVID-19 $\cdot$ Otolaryngologist $\cdot$ Rhinopharyngeal swab $\cdot$ Mannequin $\cdot$ Endoscope

Rhinopharyngeal and oropharyngeal swabs are used to test patients for COVID-19; unfortunately, the false negative rate is around $30 \%$ in symptomatic patients [1]. Consequently, it is extremely important to perform this diagnostic procedure precisely and correctly [2], but at the time of writing, the methods to train personnel in performing rhinopharyngeal swabs have not been standardized.

In the Letter to the Editor recently published by Tagliabue et al. [3], the personnel dedicated to swabs collection benefited from directly observing nasal anatomy during an endoscopy performed by an otolaryngologist, acquiring major confidence in swabs collection.

This comment refers to the article available at https://doi.org/10. 1007/s00405-020-06027-2.

Francesca Boscolo Nata

francesca.boscolonata@gmail.com

1 Otorhinolaryngology Unit, Ospedali Riuniti Padova Sud "Madre Teresa di Calcutta" Hub Covid Hospital Monselice (Padova) - ULSS, 6 Euganea, Padua, Italy

2 Laboratory Medicine Unit, Ospedali Riuniti Padova Sud "Madre Teresa di Calcutta" Hub Covid Hospital Monselice (Padova) - ULSS, 6 Euganea, Padua, Italy
We agree that performing rhinopharyngeal swabs under direct endoscopic visualization would be the ideal situation to collect precisely rhinopharyngeal secretions. However, in our opinion, this proposal has timing and financial drawbacks.

A rigid endoscope should be used to perform the procedure. Considering that two objects (the rigid endoscope and the swab) would be inserted in the nasal cavity, the procedure could result in patient's discomfort, especially if unfavourable anatomy is present. Consequently, topical anaesthesia should be performed before starting the procedure, waiting some minutes for it to take effect.

As it happens in other infectious diseases, the patient we are testing must be considered infected until proven otherwise: therefore, two health workers are potentially exposed to infection, and the endoscope must be sterilized after the procedure. The sterilization process has technical times and performing it between one patient and another further increases timing.

Consequently, both timing and costs would increase.

When performing a rhinopharyngeal swab, both pre-analytic and analytic factors, potentially jeopardizing sensitivity, should be remembered. 
Pre-analytic factors:

- Sampling method: the swab can be wrongly positioned in different nasal areas, for different timing, and in different ways (once positioned it could be maintained still or rotated by the operator)

- Viral levels are variable during the disease because cells are at different stages of their life cycle and can release the virus into the blood at different times

- Secretion amount varies in different patients, as the viral load detectable in it [4]

Analytic factors:

- Sampling is diluted with buffer solution before analysis

- Two different texts are available, one developed by the University Charitè Hospital of Berlin detecting 3 genes, the other by CDC detecting 2 genes [5]. If more genes are tested, the sensitivity increases

Consequently, an increase in sensitivity using an endoscope-guided procedure can only be supposed with the current knowledges. Moreover, studies focused on the different viral loads in the various anatomic areas (turbinates vs nasal septum vs rhinopharynx) have not been conducted; thus, it is not sure that rhinopharynx is really the best area to collect secretions for the swab.

- In our opinion, dedicated personnel could benefit in its training from the use of a mannequin that could be

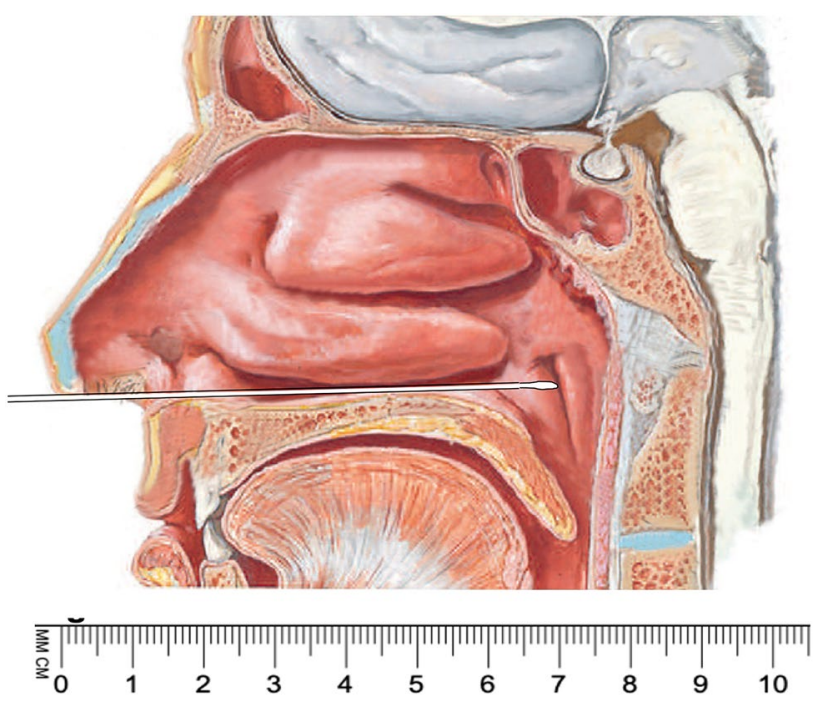

Fig. 1 Sagittal section of the nasal cavity obtained after the mannequin has been opened in the midline. It is possible to verify that the swab has been correctly positioned: it lies on the nasal floor and finally reaches the rhinopharynx, approximately $7-8 \mathrm{~cm}$ from the nasal vestibule opened on the midline to observe a sagittal section of nasal cavity. With this method, the above listed problems could be overcome. The swab collection can be simulated under the otolaryngologist's supervision: the doctor asks to the nurse to insert a swab in the nasal cavity of the mannequin, moving forward on the nasal floor until a resistance is felt (approximately 7-8 cm); when the swab is positioned, the mannequin is opened in the midline and the otolaryngologist verifies if the swab has reached the target (rhinopharynx) (Fig. 1). The procedure can be repeated until the nurse completes the learning curve and can easily and safely perform the procedure.

In our opinion, the collaboration between otolaryngologist and dedicated personnel, and the use of a mannequin, could represent a safe, time and cost saving procedure for rhinopharyngeal swab collection.

Funding None

\section{Compliance with ethical standards}

Conflict of interest The authors declare that they have not conflict of interest.

Ethical approval Not applicable.

Informed consent Non applicable.

\section{References}

1. Loeffelholz MJ, Tang YW (2020) Laboratory diagnosis of emerging human coronavirus infections - the state of the art. Emerg Microb Infect 9:747-756. https://doi.org/10.1080/22221751.2020. 1745095

2. Marty FF, Chen K, Verrill KA (2020) How to obtain a nasopharyngeal swab specimen. N Engl J Med. https://doi.org/10. 1056/NEJMvcm 2010260

3. Tagliabue M, Pietrobon G, Ugolini S, Chu F, Ansarin M (2020) Nasopharyngeal swabs during SARS-CoV-2 pandemic: a role for the otolaryngologist. Eur Arch Otorhinolaryngol. https://doi.org/ 10.1007/s00405-020-06027-2

4. To KK, Tsang OT, Leung WS et al (2020) Temporal profiles of viral load in posterior oropharyngeal saliva samples and serum antibody responses during infection by SARS-CoV-2: an observational cohort study. Lancet Infect Dis 20:565-574

5. European Centre for Disease Prevention and Control. https:// www.eurosurveillance.org/content/10.2807/ese.17.49.20334-en. Accessed 4 May 2020

Publisher's Note Springer Nature remains neutral with regard to jurisdictional claims in published maps and institutional affiliations. 